\title{
Attosecond pump-probe transition-state spectroscopy of laser-induced molecular dissociative ionization: Adiabatic versus nonadiabatic dressed-state dynamics
}

\author{
C. Lefebvre, ${ }^{1,2, *}$ T. T. Nguyen-Dang, ${ }^{1, \dagger}$ F. Dion, ${ }^{1}$ M. J. J. Vrakking, ${ }^{3}$ V. N. Serov ${ }^{2}$ and O. Atabek ${ }^{2, \ddagger}$ \\ ${ }^{1}$ Département de Chimie, Université Laval, Québec, G1K 7P4, Québec, Canada \\ ${ }^{2}$ Institut des Sciences Moléculaires d'Orsay, CNRS and UMR 8214, Bâtiment 350, Université Paris Sud, 91405 Orsay, France \\ ${ }^{3}$ Max Born Institute for Nonlinear Optics and Short Pulse Spectroscopy, Max-Born-Strasse 2A, 12489 Berlin, Germany
}

(Received 13 August 2013; published 15 November 2013)

\begin{abstract}
We discuss how a recent pump-probe study [Kelkensberg et al., Phys. Rev. Lett. 103, 123005 (2009)] of the dissociative ionization of $\mathrm{H}_{2}$, under the combined effect of a single extreme ultraviolet attosecond pulse and an intense near-infrared pulse, actually represents a transition-state spectroscopy of the strong-field dissociation step, i.e., of the (probe-pulse-)dressed $\mathrm{H}_{2}{ }^{+}$molecular ion. The way the dissociation dynamics is influenced by the duration of the near-infrared probe pulse, and by the time delay between the two pulses, is discussed in terms of adiabatic versus nonadiabatic preparation and transport of time-parametrized Floquet resonances associated with the dissociating molecular ion. Under a long probe pulse, the field-free vibrational states of the initial wave packet are transported, in a one-to-one manner, onto the Floquet resonances defined by the field intensity of the probe pulse and propagated adiabatically under the pulse. As the probe pulse duration shortens, nonadiabatic transitions between the Floquet resonances become important and manifest themselves in two respects: first, as a vibrational shake-up effect occurring near the peak of the short pulse, and second, through strong interference patterns in the fragment's kinetic energy spectrum, viewed as a function of the time delay between the pump and the probe pulses.
\end{abstract}

DOI: 10.1103/PhysRevA.88.053416

PACS number(s): $33.80 . \mathrm{Gj}, 42.50 . \mathrm{Hz}, 82.20 . \mathrm{Wt}$

\section{INTRODUCTION}

The advent of femtosecond laser pulses in the 1990s has been determinant for the development of transition-state spectroscopy and femtosecond chemistry [1]. They were basically used in pump-probe experiments [2,3], to map out, in a time-resolved manner, nuclear dynamics in molecules [4]. The production of attosecond pulses, one of the most remarkable outcomes of research done on high-harmonic generation (HHG) [5-7], now gives rise to new possibilities. First, the faster electronic motion can obviously be probed in a time-resolved manner [6,8]. Second, attosecond pulses can serve as pump pulses in pump-probe interferometric experiments, either to image electronic wave packets, as done in Ref. [9], or to give an indirect probe of nuclear motions, e.g., the laser-induced dissociation of a molecule, as found in Refs. [10] and [11], and further studied in Ref. [12] with an emphasis placed on the coherence between ionizations induced by individual pulses of an attosecond pulse train.

An important concern in these experiments is the question as to which extent the structure and dynamics of the molecules under investigation are influenced by the presence of the intense infrared (IR) laser field, the same that drives the high-harmonic generation process [13] used in the production of the attosecond XUV pulse or pulse train. Depending on the laser field's frequency and intensity regime, the dynamical and structural changes that the field can cause within the molecule are either of the type associated with the quasistatic picture or that associated with the Floquet picture. The first type of molecular restructuring, operative in the low-frequency (IR)

\footnotetext{
*Now at INRS-EMT, 1650 Boul. Lionel-Boulet, Varennes, J3X 1S2, Québec, Canada; catherine.lefebvre@emt.inrs.ca

†Thanh-Tung.Nguyen-Dang@chm.ulaval.ca

†'Osman.Atabek@u-psud.fr
}

regime, gives rise to the opportunity of controlling molecular dissociation by directly synchronizing molecular wave packets with a time-dependent potential energy barrier to dissociation (the so-called dynamical dissociation quenching (DDQ) effect [14]). For a higher frequency, in the near infrared (NIR) or the visible spectral range, the dissociation dynamics transit through laser-induced resonances defined and supported by cycled-averaged dressed electronic states [15].

In the pump-probe experiment of Ref. [10], an ultrafast XUV (pump) pulse triggers the ionization of a $\mathrm{H}_{2}$ molecule during the exposure to a longer NIR (probe) pulse, which would cause the dissociation, or the vibrational trapping, of the resulting $\mathrm{H}_{2}{ }^{+}$molecular ion depending on the delay between the two pulses. The results of the experiments, supported by complete wave-packet simulations, are stunning and pertain to the dissociation product kinetic energy release (KER) spectrum, viewed as a function of the delay between the two pulses. For a very short (7-fs FWHM) NIR probe pulse, the beating pattern impressed upon the whole KER spectrum as the delay between the pump and probe pulse is varied seems to merely reflect the vibrational content of the nuclear wave packet of the ion initially prepared by the XUV pump pulse. It carries no information on the transient restructuring of the $\mathrm{H}_{2}{ }^{+}$ion induced by the NIR field, i.e., the transition state of the laser-induced dissociation process. This was taken as the signature of a practically sudden dynamics of the laser-induced dissociation that occurs every time a nonzero probability amplitude is instantly found at the position of some "gate to dissociation" opened up by the NIR field at its almost instant onset. In contrast, a longer, 35-fs FWHM probe pulse leads to a completely different type of delay-dependent KER spectrum, reflecting a nontrivial dissociation dynamics that unfolds through laser-induced resonances within the NIR pulse.

There are many aspects of the whole interpretation that have not been addressed in Refs. [10] and [11]: How does one attribute the various structures of the $\mathrm{H}^{+}$KER spectrum to the 
different decay channels associated with laser-induced Floquet resonances [16]? To which extent are these resonances, the proper transition states of the laser-induced molecular dissociation process, identifiable with field-free vibrational states of the molecular ion and when do they differ from these? What are the signatures of the dynamical restructuring of the molecule in these transition states? In other words, can one track in detail, i.e., in a time-resolved manner, the resonance dynamics, their populations, their mixings, their time-dependent shifts, and broadenings? How does adiabaticity of the wave-packet transports through laser-induced resonances manifest itself, and how essential is nonadiabaticity to the imaging of the initial wave packet? It is the particular question of adiabatic vs nonadiabatic Floquet transition-state dynamics that will mainly retain our attention in the present paper. After a brief review of the concepts of Floquet resonance states and of adiabatic Floquet representation, to be found in Sec. III, we will illustrate, on the widespread two-state model of $\mathrm{H}_{2}{ }^{+}$[14] (recalled in Sec. II along with relevant details of the computational methodology), how the dissociation of the molecular ion, born by sudden ionization of a parent $\mathrm{H}_{2}$ molecule under the action of the XUV pump pulse, can be unraveled in terms of projections onto laser-induced resonances. This projection can be gradual or sudden (adiabatic or nonadiabatic preparation of the Floquet wave packet), depending on the delay between the probe and the pump pulses, and in terms of the subsequent evolutions of the resonances' populations, which can also be slow (adiabatic) or rapid (nonadiabatic), depending on the duration of the NIR probe pulse.

To illustrate a situation in which adiabatic Floquet state transport certainly occurs, in Sec. IV we will first consider a long, 150-fs FWHM pulse at the carrier-wave frequency corresponding to $\lambda=790 \mathrm{~nm}$, for which well-resolved features of the fragment's KER spectrum can easily be attributed to resonances that are adiabatically connected with field-free vibrational states of $\mathrm{H}_{2}{ }^{+}$. We show analytically and observe numerically that such an adiabatic Floquet dynamics gives rise to a KER spectrum that is independent of the pump-probe delay $\tau$, insofar as this is considered in the range where the two pulses do not overlap each other. In contrast, whenever nonadiabatic transitions between Floquet resonances occur within the probe pulse, the KER spectrum, viewed as a function of $\tau$ (always within the condition of nonoverlapping pump-probe pulses), exhibits interference fringes that partially reflect the coherence of the initial Franck-Condon vibrational wave packet. It is as if this coherent wave packet sees the NIR probe pulse as an open gate which merely serves to bring the wave packet out to the asymptotic and dissociative region, whereas, in fact, it encounters important nonadiabatic transitions among the Floquet resonances onto which it may project. These interference fringes were observed in the experimental spectrum of Ref. [10] for the case of the 7-fs probe pulse. Here we show that this signal of nonadiabaticity is also present in the case of the 35 -fs probe pulse, although it is not detected experimentally.

When the pump overlaps the probe pulse at an appreciable intensity, a vibrational shake-up effect is expected, as the newly born ion's state instantly projects onto high-intensity Floquet resonances that differ strongly from the field-free vibrational states. This vibrational shake-up effect will be

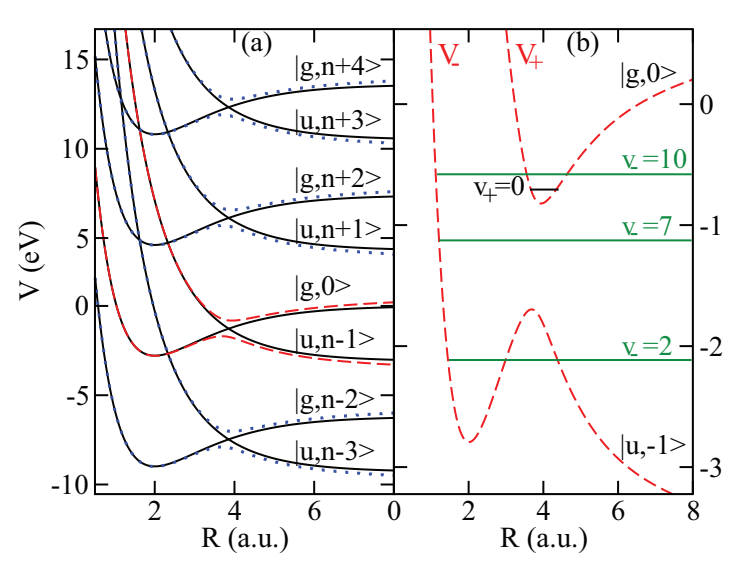

FIG. 1. (Color online) (a) Dressed potential energy curves in four Floquet blocks, in the diabatic (solid lines) and adiabatic (red dashed lines in the central Floquet block and blue dotted lines in the other Floquet blocks) representations. (b) Three shape resonance levels $\left(v_{-}=2,7,10\right)$ and a Feshbach resonance level $\left(v_{+}=0\right)$, shown on the dressed adiabatic potential energy curves, in the neighborhood of the one-photon avoided crossing of a single Floquet block.

illustrated explicitly, at the end of Sec. IV, in the case of the 35 -fs probe pulse, which gives a dynamical regime that is intermediate between adiabatic and strongly nonadiabatic Floquet resonance dynamics, represented by the cases of the 150-fs and the 7-fs FWHM probe pulses, respectively.

\section{MODEL AND METHODOLOGY}

The main results discussed in the present paper pertain to the dissociation of $\mathrm{H}_{2}{ }^{+}$under the probe NIR pulse, after its preparation in an initial nuclear wave packet by the XUV attosecond pump pulse. The two-(electronic)-state model of the molecular ion is described by the following Hamiltonian:

$$
H(t)=T_{N}+\left(\begin{array}{cc}
V_{1}(R) & 0 \\
0 & V_{2}(R)
\end{array}\right)-\mu_{12}(R) \mathcal{F}(t)\left(\begin{array}{ll}
0 & 1 \\
1 & 0
\end{array}\right)
$$

with ab initio potentials $V_{1}(R), V_{2}(R)$ associated respectively to the ground, $\left.|1\rangle=\left.\right|^{2} \Sigma_{g}\right\rangle=\left|1 \sigma_{g}\right\rangle=|g\rangle$, and first excited, $\left.|2\rangle=\left.\right|^{2} \Sigma_{u}\right\rangle=\left|1 \sigma_{u}\right\rangle=|u\rangle$, electronic states and transition dipole moment $\mu_{12}$ between them taken from [17]. Figure 1 shows these potentials dressed by $400-\mathrm{nm}$ NIR photons and their deformations due to the field-induced couplings $\mathcal{F}_{0} \mu_{12} / 2$ between these dressed potentials. Wave-packet propagations are made in one dimension, along the radial coordinate $R$ only, neglecting molecular rotations that occur on a much longer time scale. The nuclear kinetic energy $T_{N}$ in Eq. (1) is thus purely radial. Also, since dissociation occurs mainly along the direction of the field polarization, we have considered perfectly aligned molecules in the simulations.

Calculations use a NIR pulse of the form

$$
\overrightarrow{\mathcal{F}}(t)=\vec{\epsilon} \mathcal{F}_{0} g(t-\tau) \cos [\omega(t-\tau)+\theta],
$$

where $\vec{\epsilon}$ is the linearly polarized laser polarization vector, $\mathcal{F}_{0}$ is the field amplitude, $\omega=2 \pi c /(\lambda=790 \mathrm{~nm})$ is the carrier-wave frequency, and $\theta$ is the carrier-envelope phase, here fixed to $\theta=0$. The field amplitude $\mathcal{F}_{0}$ is given a value 
corresponding to a peak intensity $I=3 \times 10^{13} \mathrm{~W} / \mathrm{cm}^{2},(I \propto$ $\left.\mathcal{F}_{0}^{2}\right)$. The envelope $g(t-\tau)$ is a Gaussian function centered at $\tau$ and is given by

$$
g(t-\tau)=e^{-\left[2 \sqrt{\ln 2}(t-\tau) / T_{\mathrm{FWHM}}\right]^{2}},
$$

with $T_{\text {FWHM }}$ the FWHM of the electric field pulse duration. We will consider three values of $T_{\mathrm{FWHM}}$ in the following, representing a long pulse $T_{\mathrm{FWHM}}=150 \mathrm{fs}$, a short pulse $T_{\mathrm{FWHM}}=35 \mathrm{fs}$, and an ultrashort, few-cycle pulse $T_{\mathrm{FWHM}}=7 \mathrm{fs}$. To the two probe pulse durations considered in the experiments of Ref. [10] (the last two values of $T_{\text {FWHM }}$ ), we have thus added the case of a much longer pulse, representing the most adiabatic situation and better approaching the continuous wave (cw) limit so as to allow a clear, nonambiguous assignment of lines in the calculated fragment kinetic energy spectra.

In these calculations, we launch the wave-packet propagation procedure at a time $t=0$, corresponding to the onset of ionization of the parent molecule by the XUV attosecond (as) pulse, so that $\tau$ represents the delay between the pump and the probe pulses. The initial state is then taken to be a Franck-Condon (FC) wave packet, i.e., it is the ground vibrational state of $\mathrm{H}_{2}, \chi_{0}^{\mathrm{H}_{2}}(R)$, promoted vertically onto the ground manifold [potential $V_{1}(R)$ above] of the molecular ion. The wave-packet propagation is then done by solving the time-dependent Schrödinger equation (TDSE):

$$
\begin{aligned}
& i \hbar \frac{\partial}{\partial t}\left(\begin{array}{l}
\chi_{1}(R, t) \\
\chi_{2}(R, t)
\end{array}\right) \\
& =\left\{T_{N}+\left(\begin{array}{cc}
V_{1}(R) & 0 \\
0 & V_{2}(R)
\end{array}\right)-\mu_{12}(R) \mathcal{F}(t)\left(\begin{array}{ll}
0 & 1 \\
1 & 0
\end{array}\right)\right\} \\
& \times\left(\begin{array}{l}
\chi_{1}(R, t) \\
\chi_{2}(R, t)
\end{array}\right) \text {. }
\end{aligned}
$$

The functions $\chi_{j}(R, t), j=1,2$, are the nuclear amplitudes associated with the two electronic states of the molecule, i.e.,

$$
|\Psi(R, t)\rangle=\chi_{1}(R, t)|1\rangle+\chi_{2}(R, t)|2\rangle .
$$

The TDSE (4) is solved numerically, using a stroboscopic representation of the time-evolution operator

$U(t, 0)=U\left(t=t_{n}, t_{n-1}\right) U\left(t_{n-1}, t_{n-2}\right) U\left(t_{n-2}, t_{n-3}\right) \ldots . U\left(t_{1}, t_{0}\right)$,

with the propagator $U\left(t_{n}, t_{n-1}\right)$ over a short-time slice $\left[t_{n-1}, t_{n}\right]$, computed by a procedure that has been thoroughly documented in previous works [16]. For the inner part of the nuclear wave functions, $\chi_{j}(R, t), j=1,2$, defined over the region of the potentials where Hellmann-Feynman forces are nonzero, numerical propagation on a grid uses the standard third-order split-operator algorithm [18], while for the outer part of the wave functions, defined over the asymptotic region of the potential energy curves, where these curves level off each to a constant value, an analytical propagation can be made since the transition dipole moment behaves as a linear function of $R$. This analytical propagation is done by projecting the asymptotic nuclear wave functions on Volkov-type states $[19,20]$. From the accumulated asymptotic part of the wave packet expressed in momentum space, the relative kinetic energy of dissociation on each of the two field-free channels $|g\rangle$ and $|u\rangle$ is calculated through

$$
S_{g(u)}\left(E, t_{f}\right)=s_{g(u)}\left(k=+\sqrt{2 M E}, t_{f}\right),
$$

where

$$
s_{g(u)}\left(k, t_{f}\right)=\left|\left\langle k \mid \chi_{g(u)}^{A}\left(t_{f}\right)\right\rangle\right|^{2}
$$

is the momentum content of $\chi_{g(u)}^{A}$, the asymptotic nuclear wave packet of channel $g$ (or $u$ ), at the final time $t_{f}$. $|k\rangle$ denotes the plane wave $\langle R \mid k\rangle \propto e^{i k R}$ of wave vector $k, M$ is the reduced mass of the molecular ion, and $E$ is the kinetic energy. The total relative kinetic energy spectrum $S\left(E, t_{f}\right)$ is the sum of $S_{g}\left(E, t_{f}\right)$ and $S_{u}\left(E, t_{f}\right)$. The final time $t_{f}$ depends on the duration of the NIR pulse $\left(T_{\text {FWHM }}\right)$ and on the pump-probe delay $\tau$. We will focus on the way $S_{g}(E)$ and $S_{u}(E)$ vary with $\tau$ and discuss this dependence in terms of Floquet resonances and dynamical regimes. Relevant concepts and relations derived from Floquet theory are reviewed in the next section. Note that no calculation is done directly in the Floquet representation, which is used for interpretation only.

\section{LASER-INDUCED (FLOQUET) RESONANCES}

\section{A. Floquet theory: A short review}

If the Hamiltonian $H(t+T)=H(t)$ is time periodic, which would be the case if $g(t)$ in Eq. (2) is a constant representing a cw field for which $T=2 \pi / \omega$, then the Floquet theorem [21-23] states that the TDSE [Eq. (4)] admits particular solutions of the form

$$
\left|\Psi_{\mathcal{E}}(R, t)\right\rangle=e^{-i \mathcal{E} t / \hbar}\left|\Phi_{\mathcal{E}}(R, t)\right\rangle
$$

or, equivalently,

$$
\left(\begin{array}{l}
\chi_{1}(R, t) \\
\chi_{2}(R, t)
\end{array}\right)=e^{-i \mathcal{E} t / \hbar}\left(\begin{array}{l}
\phi_{1}^{(\mathcal{E})}(R, t) \\
\phi_{2}^{(\mathcal{E})}(R, t)
\end{array}\right),
$$

with $\phi_{j}^{(\mathcal{E})}(R, t),(j=1,2)$ (the two components of $\left|\Phi_{\mathcal{E}}(R, t)\right\rangle$ ) time-periodic functions. These Floquet states $\left|\Phi_{\mathcal{E}}(R, t)\right\rangle$ are eigenfunctions of the so-called Floquet Hamiltonian,

$$
K=\left[H(t)-i \hbar \frac{\partial}{\partial t}\right],
$$

with associated eigenvalue $\mathcal{E}$ called the quasienergy of the Floquet state:

$$
K\left|\Phi_{\mathcal{E}}(R, t)\right\rangle=\mathcal{E}\left|\Phi_{\mathcal{E}}(R, t)\right\rangle .
$$

Note that the Floquet Hamiltonian is defined in an extended space, the direct product of the usual molecular Hilbert space $(\mathcal{H})$ and the space $L^{2}$ of periodic functions of $t \in[0, T]$ [24-26]. We note that since they are time periodic, the functions $\phi_{j}^{(\mathcal{E})}(R, t)$ can be Fourier expanded as

$$
\phi_{j}^{(\mathcal{E})}(R, t)=\sum_{n=-\infty}^{+\infty} e^{i n \omega t} \psi_{j, n}^{(\mathcal{E})}(R),
$$

so that one gets from Eq. (10) an infinite set of coupled, timeindependent equations for the nuclear wave-function Floquet components $\psi_{j, n}^{(\mathcal{E})}(R)$ [whenever possible, the superscript ( $)^{(\mathcal{E})}$ 
will be dropped from now on]:

$$
\begin{aligned}
& {\left[T_{N}+V_{1}(R)+n \hbar \omega\right] \psi_{1, n}(R)} \\
& \quad-\frac{1}{2} \mathcal{F}_{0} \mu_{12}(R)\left[\psi_{2, n-1}(R)+\psi_{2, n+1}(R)\right]=\mathcal{E} \psi_{1, n}(R), \\
& {\left[T_{N}+V_{2}(R)+n \hbar \omega\right] \psi_{2, n}(R)} \\
& \quad-\frac{1}{2} \mathcal{F}_{0} \mu_{21}(R)\left[\psi_{1, n-1}(R)+\psi_{1, n+1}(R)\right]=\mathcal{E} \psi_{2, n}(R) .
\end{aligned}
$$

The structure of these equations is such as to define a parity selection rule: Once a parity of the Fourier indices ("number of photons") $n$ associated with the state $|1\rangle$ is chosen, the nuclear amplitudes $\psi_{1, n}$ supported by this state, dressed by " $n$ photons" (i.e., $\left.V_{1}(R)+n \hbar \omega\right)$, are coupled only to the amplitudes $\psi_{2, n^{\prime}}$ supported by the state $|2\rangle$ with a number $n^{\prime}$ of opposite parity.

\section{B. Dressed molecule diabatic and adiabatic pictures}

In the limit $\mathcal{F}_{0} \rightarrow 0$, Eqs. (12) become decoupled and define the diabatic Floquet representation, and $\psi_{j, n}(R)$ is the amplitude (wave function) of the $n$-photon dressed (diabatic) $j$ th channel in the Floquet $\left|\Phi_{\mathcal{E}}(R, t)\right\rangle$ eigenstate. The field interaction $\mathcal{F}_{0} \mu_{12}(R)$ couples the diabatic $n$-photon-dressed $j$ th channel amplitudes $\psi_{j, n}(R)$ together. An illustration of the photon-dressed diabatic potentials (solid black lines) is given in Fig. 1(a), showing explicitly four Floquet blocks defined by the dressing of the molecule by a $\lambda=400 \mathrm{~nm}$ field [27]. The reference (central) block therein corresponds to a single-photon absorption described by the crossing of two potentials curves: $V_{1}(R)$ and $V_{2}(R)-\hbar \omega$. The other blocks correspond to multiphoton processes $(-2$ and -3 for two- and three-photon absorptions; +1 and +2 for one- and two-photon emissions). Diagonalizing the potential part of the full Floquet Hamiltonian matrix defined by Eq. (12), i.e., including the diabatic-channel couplings $\left[\mathcal{F}_{0} \mu_{12}(R)\right]$, one gets an equivalent picture, called the adiabatic Floquet scheme, with channel crossings in the diabatic representation becoming avoided crossings where adiabatic amplitudes $\psi_{j, n}^{\text {(adia) }}(R)$ are now coupled together by sharply localized field-induced (spatial) nonadiabatic couplings. This (R-) adiabatic frame, characterized by avoided curve crossings [16,28], is represented in Fig. 1(a) by the dashed red lines in the central block and by dotted blue lines in the other Floquet blocks (neglecting third-order couplings). The adiabatic potential energy curves in Fig. 1 are obtained by diagonalization of a truncated Floquet Hamiltonian matrix. Four blocks are retained in constructing panel (a) to highlight pathways of the main multiphoton processes discussed hereafter. Dominant single-photon processes could approximatively be described by the diagonalization of a truncated Hamiltonian limited to the single central block as represented in panel (b).

\section{Laser-induced resonances}

In the presence of the laser, the field-free bound vibrational levels of the electronic state $|1\rangle$, labeled by $v$, become multichannel Floquet resonances which are obtained by imposing the so-called Siegert outgoing boundary conditions [29] to the solution of Eqs. (12):

$$
\lim _{R \rightarrow \infty} \psi_{j, n}(R) \propto e^{+i k_{j n} R},
$$

which leads to discretized complex eigenenergies labeled by $\tilde{v}$ such that

$$
\mathcal{E}_{\tilde{v}}=\operatorname{Re}\left[\mathcal{E}_{\tilde{v}}\right]-i \Gamma_{\tilde{v}} / 2, \quad\left(\Gamma_{\tilde{v}}>0\right),
$$

with $\quad k_{j n}^{\tilde{v}} \equiv \operatorname{Re}\left[k_{j n}^{\tilde{v}}\right]-i \operatorname{Im}\left[k_{j n}^{\tilde{v}}\right]=\sqrt{2 M\left[\mathcal{E}_{\tilde{v}}-\epsilon_{j}-n \hbar \omega\right]}$, $\operatorname{Re}\left[k_{j n}^{\tilde{v}}\right]$ and $\operatorname{Im}\left[k_{j n}^{\tilde{v}}\right]>0$, and $\epsilon_{j}=\lim _{R \rightarrow \infty} V_{j}(R)$. These Floquet resonances can be said to belong to one of the two following categories, depending on the adiabatic potential they are associated with $[27,30,31]$. The resonances supported by the lower adiabatic potential $V_{-}(R)$ in the Floquet block shown in Fig. 1(b) are of shape type, whereas those belonging to the upper adiabatic potential $V_{+}(R)$ are of Feshbach type. The shape resonances, labeled by $v_{-}$, of complex energies $\operatorname{Re}\left[\mathcal{E}_{v_{-}}\right]-i \Gamma_{v_{-}} / 2$ are either (i) long-lived tunnel resonances (the lowest-energy ones), which are well protected against dissociation by the field-induced potential barrier created at the one-photon crossing, or (ii) short-lived above-the-barrier resonances. The stronger the field, the lower the potential barrier will be, leading to shorter lifetimes and more efficient dissociation through these resonances. This is the bond-softening (BS) mechanism [16]. Three typical examples of shape resonances are illustrated in Fig. 1(b): one long-lived, the $v_{-}=2$ level, at low energies; and two short-lived, the $v_{-}=7$ level, at relatively low energies above the potential barrier; and one at higher energies, the $v_{-}=10$ level. The Feshbach resonances, labeled by $v_{+}$with corresponding energies $\operatorname{Re}\left[\mathcal{E}_{v^{+}}\right]-i \Gamma_{v^{+}} / 2$, are in principle long lived. Their behavior with respect to the field strength is opposite to that of the shape resonances: the stronger the field, the lower will the residual nonadiabatic couplings be, resulting in a stabilization of the molecule. The effect of these resonances on the dissociation dynamics is thus opposite to the BS mechanism. The mechanism involving these resonances is called vibrational trapping (VT) or bond-hardening (BH) [16]. The position of $v_{+}=0$ is given as an example in Fig. 1(b), again for the dressing of the molecular ion by a photon of wavelength $\lambda=400 \mathrm{~nm}$.

\section{Floquet representation: cw field}

The solutions of the Floquet eigenvalue equation possess a periodicity property which results from the time periodicity of the Hamiltonian $H(t)$ [24,31]: If $\mathcal{E}_{\gamma}$ is a quasienergy, i.e., an eigenvalue of the Floquet Hamiltonian $K$ associated with an eigenstate $\left|\Phi_{\mathcal{E}_{\gamma}}(R, t)\right\rangle=e^{-i \mathcal{E}_{\gamma} t / \hbar}\left\{\phi_{1}^{\left(\mathcal{E}_{\gamma}\right)}(R, t)|1\rangle+\right.$ $\left.\phi_{2}^{\left(\mathcal{E}_{\gamma}\right)}(R, t)|2\rangle\right\}$, then $\mathcal{E}_{\gamma, \kappa}=\mathcal{E}_{\gamma}+\kappa \hbar \omega, \quad \kappa \in \mathbb{Z}$, is also an eigenvalue, i.e., it also belongs to the Floquet energy spectrum. The eigenvector associated with this eigenvalue is obtained by multiplying $\left|\Phi_{\mathcal{E}_{\gamma}}(R, t)\right\rangle$ with $e^{i \kappa \omega t}$, defining an equivalence relationship between Floquet states. The indices $n$ labeling the Fourier components $\psi_{j, n}^{\left(\mathcal{E}_{\gamma}\right)}(R)$ are shifted accordingly by $-\kappa$, i.e., $\psi_{j, n}^{\left(\mathcal{E}_{\gamma}, \kappa\right)}(R)=\psi_{j, n-\kappa}^{\left(\mathcal{E}_{\gamma}\right)}(R)$. As a consequence, Floquet quasienergies are defined modulo an integer multiple of $\hbar \omega$, and a Floquet state, and its energy are identified by two indices: The index $\gamma$ identifies the state's equivalence class and the index $\kappa$ defines the Brillouin zone to which the state belongs. In the present problem, $\gamma$ is generally a continuous index labeling continuum half-scattering states. Within this continuum, a 
countable number of resonance states (of complex energies $\mathcal{E}_{\tilde{v}}$ ) are found, and will be labeled rather by a discrete index $\tilde{v}$.

The Floquet states $\left|\Phi_{\mathcal{E}_{\gamma}, \kappa}(R, t)\right\rangle$, resonances, and pure continuum states, taken altogether (all Brillouin zones), form a complete basis in the extended Hilbert space $\mathcal{H} \otimes L^{2}$. We are rather interested in the state evolved, in the physical, molecular Hilbert space $\mathcal{H}$, from some initial state $\left|\Psi_{0}\left(R, t_{0}\right)\right\rangle=\chi_{0,1}(R)|1\rangle+\chi_{0,2}(R)|2\rangle$ prepared at some time $t_{0}[24,32]$. This expression, of the time-dependent wave packet evolving from $\left|\Psi_{0}\left(R, t_{0}\right)\right\rangle$, reads [note the simplified notations $\left.\phi_{j}^{(\gamma)}(R, t) \leftrightarrow \phi_{j}^{\left(\mathcal{E}_{\gamma}, \kappa=0\right)}(R, t)\right]:$

$$
\begin{aligned}
& U\left(t, t_{0}\right)\left|\Psi_{0}(R)\right\rangle \\
& \quad=\bigvee_{\gamma} Z_{\Psi_{0}}^{(\gamma)}\left|\Phi_{\mathcal{E}_{\gamma}, \kappa=0}(R, t)\right\rangle \\
& =\bigvee_{\gamma} Z_{\Psi_{0}}^{(\gamma)} e^{-i \mathcal{E}_{\gamma}\left(t-t_{0}\right) / \hbar}\left\{\phi_{1}^{(\gamma)}(R, t)|1\rangle+\phi_{2}^{(\gamma)}(R, t)|2\rangle\right\},
\end{aligned}
$$

where

$$
Z_{\Psi_{0}}^{(\gamma)}=\sum_{\kappa=-\infty}^{+\infty} e^{i \kappa \omega t_{0}}\left[\left\langle\psi_{1, \kappa}^{\left(\mathcal{E}_{\gamma}\right)} \mid \chi_{0,1}\right\rangle+\left\langle\psi_{2, \kappa}^{\left(\mathcal{E}_{\gamma}\right)} \mid \chi_{0,2}\right\rangle\right],
$$

defined at $t_{0}$, represents the projection of the initial state (with the two nuclear amplitudes $\chi_{0,1(2)}$ associated with the two electronic states $|1\rangle$ and $|2\rangle$ ) onto the Floquet states of the class identified by $\gamma$, in all the Brillouin zones.

The sum integral in Eq. (15) implies a sum over all the resonances and an integral over the rest of the continuum. It is desirable to concentrate on the contributions of the resonances alone, which can be expressed by the action of an appropriate projection operator $\mathcal{Q}$ on the time-dependent wave packet, so that

$$
\begin{aligned}
& \mathcal{Q} U\left(t, t_{0}\right)\left|\Psi_{0}(R)\right\rangle \\
& \quad=\sum_{\tilde{v}} Z_{\Psi_{0}}^{(\tilde{v})} e^{-i \mathcal{E}_{\tilde{v}}\left(t-t_{0}\right) / \hbar}\left\{\phi_{1}^{(\tilde{v})}(R, t)|1\rangle+\phi_{2}^{(\tilde{v})}(R, t)|2\rangle\right\} .
\end{aligned}
$$

\section{E. Adiabatic Floquet representation}

Rigorously, the above Floquet representation is valid only when the field is periodic (cw field). In reality, we have a time-dependent amplitude $\mathcal{F}_{0}(t)\left[\mathcal{F}_{0}(t)=\mathcal{F}_{0} g(t-\tau)\right.$ in Eq. (2)] that modulates the field oscillations at the frequency $\omega$. If the amplitude modulation is slow, the Floquet ansatz can still be applied to the TDSE within some time interval $[\bar{t}-\Delta t, \bar{t}+\Delta t], \Delta t \ll 2 \pi / \omega$ around a given time $\bar{t}$. If the variations of the pulse envelope over $2 \Delta t$ can be neglected but still ensure that some oscillations of the high-frequency carrier wave do occur during that time interval, then the laser field $\mathcal{F}(t)$ can be considered of constant amplitude therein, i.e., it can be written $\mathcal{F}(t)=\overline{\mathcal{F}}_{0} \cos \omega t$ with $\overline{\mathcal{F}}_{0}:=\mathcal{F}_{0}(\bar{t})$. The eigenstates, $\left|\Phi_{\mathcal{E}_{\tilde{v}}, K}^{(\bar{t})}(R, t)\right\rangle$, of the instantaneous Floquet Hamiltonian $K^{\{\bar{t}\}}(t)=H^{\{\bar{t}\}}(t)-i \hbar \frac{\partial}{\partial t}$ associated with this local periodic field defines a basis for the expansion of the actual wave packet of the system as it evolves during this time interval from some initial condition. Imagine now that the laser pulse duration is divided up into $\mathcal{N}_{t}$ time slices of width $\Delta t_{n}$ centered on $\bar{t}_{n}, n=0,1,2, \ldots \mathcal{N}_{t}$. If the actual time evolution across the full pulse width is essentially the transport of a single Floquet resonance state or a group of quasidegenerate Floquet states from one time slice to another, then the dynamics is said (time-) adiabatic. This definition represents the extension, to Floquet states, of the concept of adiabatic transport of eigenstates of the time-dependent Hamiltonian as expressed by the celebrated adiabatic theorem $[33,34]$. Its precise formulation within Floquet theory has been given in different forms by many authors [24,35-37]. In this case, assuming that all Floquet levels are nondegenerate, Eq. (17) becomes, in the limit $\Delta t_{n} \rightarrow 0, \forall n$,

$$
\begin{aligned}
& \mathcal{Q} U\left(t, t_{0}\right)\left|\Psi_{0}(R)\right\rangle \\
& \quad=\sum_{\tilde{v}} Z_{\Psi_{0}}^{(\tilde{v})} e^{-i / \hbar \int_{t_{0}}^{t} d t^{\prime} \mathcal{E}_{\tilde{v}}\left(t^{\prime}\right)}\left\{\phi_{1}^{(\tilde{v})}(R, t)|1\rangle+\phi_{2}^{(\tilde{v})}(R, t)|2\rangle\right\},
\end{aligned}
$$

where $Z_{\Psi_{0}}^{(\tilde{v})}$ is as given in Eq. (16), but with the Floquet states' Fourier components $\psi_{j, n^{\prime}}^{\left(\mathcal{E}_{\tilde{v}}\right)}$ defined by the instant field amplitude at $\bar{t}=t$. This expression is to be modified to describe the transport of a group of degenerate Floquet states, incorporating, among other things, possible non-Abelian geometrical phase effects $[34,35,38]$. To bring out the contrast between adiabatic and nonadiabatic dynamics, it is sufficient to refer to Eq. (18) as representing a pure adiabatic case.

In the adiabatic situation, not only do properties of the resonances vary smoothly in time, as the slowly varying field envelope modulates the adiabatic energy barrier height and width and the energy gap at the main (one-photon) avoided crossing of the dressed potentials, but resonances (or degenerate groups of these) are also transported smoothly, in a one-to-one manner, from one time slice to another. Thus, discarding some extreme cases involving so-called exceptional points corresponding to specific laser parameters inducing resonance coalescence $[39,40]$, if the fieldmolecule interaction is switched on smoothly, then an initial eigenstate of the field-free molecule, say a vibrational state $\chi_{v}(R)$ supported by the electronic ground state $|1\rangle$, will be transported adiabatically onto a single resonance and will remain in this resonance (whose properties change slowly with time) at all subsequent times until the end of the pulse. This means $\left|\Psi_{v, \kappa=0},\left(R, t_{0}\right)\right\rangle=\chi_{v}(R)|1\rangle, \quad \mathcal{E}_{v, \kappa=0}\left(t_{0}\right)=$ $\epsilon_{v}, \quad \psi_{1, n}^{(v)}=\chi_{v}(R) \delta_{n, 0}, \quad \psi_{2, n}^{(v)}=0, \quad$ and $\quad Z_{\Psi_{0}}^{\tilde{v}}=\delta_{\tilde{v}, v}, \quad$ and Eq. (18) reduces to

$$
\begin{aligned}
& \mathcal{Q} U\left(t, t_{0}\right)\left[\chi_{v}(R)|1\rangle\right] \\
& \quad=e^{-i / \hbar \int_{t_{0}}^{t} d t^{\prime} \mathcal{E}_{v}\left(t^{\prime}\right)}\left\{\phi_{1}^{(v)}(R, t)|1\rangle+\phi_{2}^{(v)}(R, t)|2\rangle\right\} .
\end{aligned}
$$

We will also encounter situations where the field-molecule interaction is switched on suddenly at a time within the pulse width when a noticeable field intensity is already attained, defining resonance states that already differ markedly from field-free molecular eigenstates. Although the preparation of the molecule in an instantaneous Floquet wave packet is sudden in this case, subsequent evolution of each component of this wave packet, i.e., of Floquet resonances, may be adiabatic within the remaining part of the laser pulse. Equation (18) then applies as such, without the reduction of the sum on its right-hand side to a single term, as shown in Eq. (19), even if $\left|\Psi_{0}\left(R, t_{0}\right)\right\rangle=\chi_{v}(R)|1\rangle$. 
It is useful to infer from Eqs. (17), (18), and (19) the corresponding expressions of the amplitude of probability for producing fragments at a given relative kinetic energy. Each resonance (Floquet) state in the central Brillouin zone (identified by $\tilde{v}$ ) will give rise to an amplitude of probability $f_{\tilde{v}}^{(j)}(E)$ for finding the system dissociated on channel $|1\rangle$ or $|2\rangle$ with a relative energy $E$, typically of the form

$$
f_{\tilde{v}}^{(j)}(E)=\sum_{n} a_{j n}^{(\tilde{v})} \mathcal{L}_{\zeta_{j n}^{(\tilde{v})}}\left[E-\left(\operatorname{Re}\left[\mathcal{E}_{\tilde{v}}\right]-\epsilon_{j}-n \hbar \omega\right)\right],
$$

$\mathcal{L}_{w}$ being the Lorentzian (centered at $\operatorname{Re}\left[\mathcal{E}_{\tilde{v}}\right]+\epsilon_{j}+n \hbar \omega$ ),

$$
\mathcal{L}_{w}(x)=2 \pi \frac{w}{x^{2}+w^{2}},
$$

with $w$ its partial width as obtained from the asymptotic amplitudes of resonance wave-function components associated with the dressed channel $(j, n)$ [41]. The partial widths are actually proportional to the branching ratios of these channels and, in the case of nonoverlapping neighboring resonances, their sum is nothing but $\Gamma_{\tilde{v}}$. The relative weight in the amplitude $f_{\tilde{v}}^{(j)}(E)$ of the Lorentzian is given by $a_{j n}^{(\tilde{v})}$, proportional to the corresponding branching ratio. A wave packet represented by Eq. (18) will then lead to a KE distribution that is the absolute square of the following complex amplitude:

$$
f^{(j)}(E)=\sum_{\tilde{v}} Z_{\Psi_{0}}^{(\tilde{v})} e^{-i / \hbar \int_{t_{0}}^{t_{f}} d t^{\prime} \mathcal{E}_{\tilde{v}}\left(t^{\prime}\right)} f_{\tilde{v}}^{(j)}(E),
$$

$t_{f}$ being a time marking the end of the NIR pulse.

\section{RESULTS AND DISCUSSION}

\section{A. Long NIR pulse: Adiabatic Floquet dynamics}

Figure 2 shows, for the case $T_{\mathrm{FWHM}}=150 \mathrm{fs}$, the fragment relative kinetic energy spectrum for four values of the delay $\tau$ between the XUV pump pulse and the long NIR probe pulse. This pulse is shown explicitly in panel (a). Its position defines the zero of the delay scale shown on the abscissa. The position of the XUV pulse $\tau$ is thus measured with respect to the center of the NIR pulse. In conformity with the convention adopted in Ref. [10], this $\tau$ scale is defined such that $\tau>\tau_{0}>0$ for a pump (XUV) pulse preceding the probe (NIR) pulse. We have defined, on the delay scale, a point denoted $\tau_{0}$ marking the effective onset of the NIR pulse so that any value of $\tau$ preceding this point can be considered, for all practical purposes, a situation where the NIR field is off. Note that since this pulse is represented by a symmetric Gaussian envelope function in the present calculations, this point can only be estimated here (for example, $\tau_{0} \simeq 200 \mathrm{fs}$ for the case of the $T_{\text {FWHM }}=150 \mathrm{fs}$ pulse) and the NIR pulse effective full width is $\simeq 2 \tau_{0}$. It is important to distinguish this delay scale from the scale of the wave packet propagation time $t$, which is defined such that the XUV pulse comes (and creates the molecular ion) at $t=0$, while the NIR pulse is centered at $t=+\tau$. The molecule encounters this NIR pulse at $t=t_{0}$. We now look at the details of the spectrum for different ranges of the XUV-NIR delay $\tau$.

\section{Pump pulse preceding probe pulse}

In this case the molecular ion, prepared in a FC wave packet by the pump pulse, experiences the whole NIR pulse and

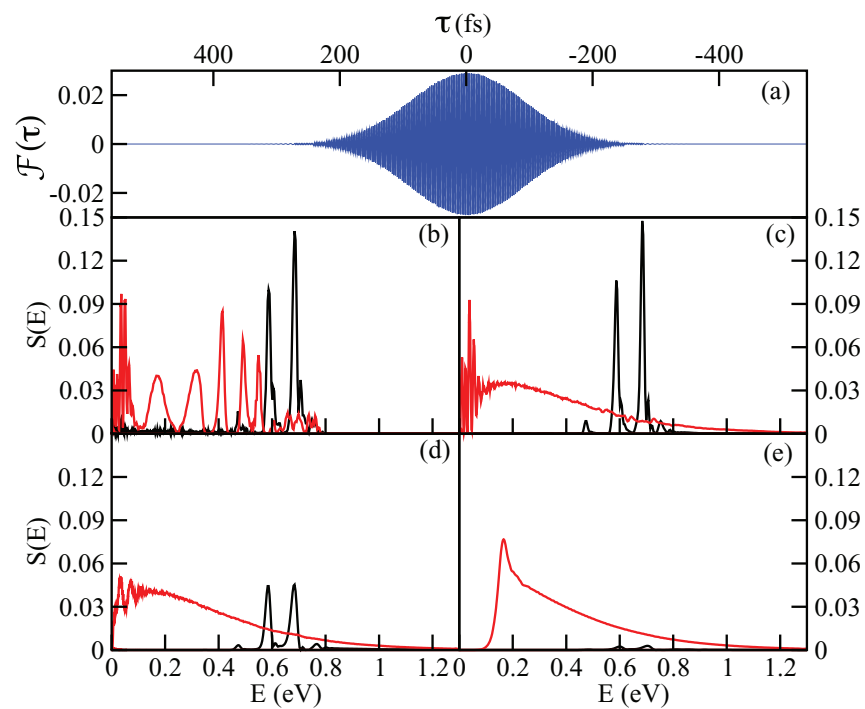

FIG. 2. (Color online) Fragment relative kinetic energy spectrum for four values of the delay $\tau$ between the XUV pump pulse and the $T_{\text {FWHM }}=150$ fs NIR probe pulse $\left(\lambda=790 \mathrm{~nm}, I=3 \times 10^{13} \mathrm{~W} / \mathrm{cm}^{2}\right)$, shown explicitly in panel (a), which also defines the delay $(\tau)$ scale. In panel (b), $\tau=+561 \mathrm{fs}$; in panel (c), $\tau=+61 \mathrm{fs}$; in panel (d), $\tau=$ $0 \mathrm{fs}$, and in panel (e), $\tau=-122 \mathrm{fs}$. In all panels, the spectrum traced out in red is associated with dissociation on the channel $|u, n-1\rangle$, and the one traced in black is for dissociation on the channel $|g, n-2\rangle$.

vibrational states $v$ are adiabatically transported onto Floquet resonances defined by the instantaneous field intensity of the NIR pulse, i.e., one has here both adiabatic branching of resonances and adiabatic evolution of resonance once created. The time $t_{0}$ in Eq. (18) corresponds to the onset of the NIR pulse $\left(\tau_{0}\right.$, on the delay scale defined above), i.e., $t_{0}=\tau-\tau_{0}$, and

$$
Z_{\Psi_{0}}^{(\tilde{v})}=\sum_{v} c_{v}^{F C} e^{-i / \hbar \epsilon_{v}\left(\tau-\tau_{0}\right)} \delta_{v, \tilde{v}}
$$

$\left(c_{v}^{F C}=\left\langle\chi_{0}^{\mathrm{H}_{2}} \mid \chi_{v}\right\rangle\right)$, in this case, so that Eq. (20b) becomes ( $t_{f}=\tau+\tau_{0}$, the end of the NIR pulse)

$$
f^{(j)}(E)=\sum_{v} c_{v}^{F C} e^{-i / \hbar\left[\epsilon_{v}\left(\tau-\tau_{0}\right)+\int_{\tau-\tau_{0}}^{\tau+\tau_{0}} d t^{\prime} \mathcal{E}_{v}\left(t^{\prime}\right)\right]} f_{v}^{(j)}(E),
$$

with $j=g, u$, and the KE spectrum is of the form

$$
\begin{aligned}
S_{j}(E)= & \left|f^{(j)}(E)\right|^{2} \\
= & \sum_{v} \sum_{v^{\prime}}\left(c_{v}^{F C}\right)^{*}\left(c_{v^{\prime}}^{F C}\right) \\
& \times e^{-i / \hbar\left\{\left(\epsilon_{v^{\prime}}-\epsilon_{v}\right)\left(\tau-\tau_{0}\right)+\int_{\tau-\tau_{0}}^{\tau+\tau_{0}} d t^{\prime}\left[\mathcal{E}_{v^{\prime}}\left(t^{\prime}\right)-\mathcal{E}_{v}\left(t^{\prime}\right)\right]\right\}} \\
& \times\left[f_{v^{\prime}}^{(j)}\right]^{*}(E) f_{v}^{(j)}(E),
\end{aligned}
$$

which shows that the KE spectrum exhibits a strong nontrivial dependance on the XUV-NIR delay $\tau$, with characteristic periodic interference fringes arising from the phase factor $e^{-i / \hbar\left[\left(\epsilon_{v^{\prime}}-\epsilon_{v}\right)\left(\tau-\tau_{0}\right)\right]}$ in Eq. (23) only if some amplitudes $f_{v}^{(j)}(E)$ overlap each other.

Panel (b) of Fig. 2 shows the KE spectrum obtained at $\tau=+561$ fs. The lower-energy vibrational states of the molecular ion are transported onto resonances supported by the lower adiabatic potential and give rise to dissociation (from 

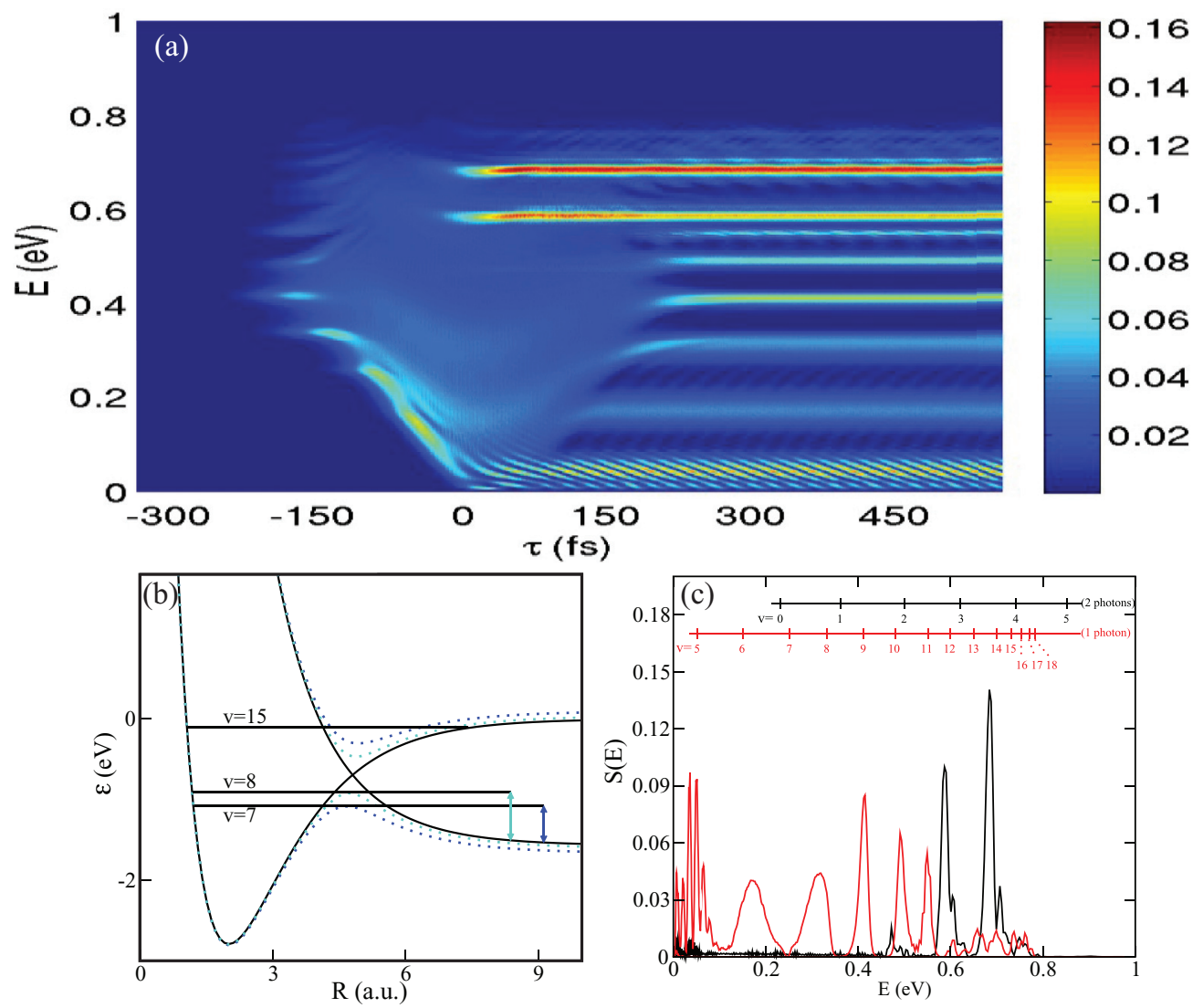

FIG. 3. (Color online) (a) Fragment relative kinetic energy spectrum viewed as a function of the delay $\tau$ between the XUV pump and the $T_{\mathrm{FWHM}}=150 \mathrm{fs}$ NIR $(\lambda=790 \mathrm{~nm})$ probe pulse. Panel (b) illustrates the principle used for the assignment of the KE spectrum's features to laser-induced resonances connected to field-free $v$ states, as shown in panel (c) for the spectrum taken at $\tau=+561 \mathrm{fs}$.

each of these resonances) by absorption of one or two photons following the BS mechanism. The positions of the peaks in the KER spectrum reflect those of corresponding resonances supported by the well of the $|g, n\rangle$ potential energy curve, with respect to the asymptote of the $|u\rangle$ channel dressed by $n-1$ photons. We have been able to make a detailed assignment of the peaks in terms of the field-free vibrational energy levels $v$, giving due allowance to Stark shift and broadening of these as they denote resonances in the presence of the field. This assignment is shown in Fig. 3(c). The resonances associated with the states $v \geqslant 5$ give rise to dissociation on the $|u, n-1\rangle$ channel, and the peaks of the fragments' kinetic energy spectrum associated with this channel (traced in red lines) are sufficiently well resolved to allow, for most of them, a one-to-one assignment of the peak to a $v$ state. The shape resonances corresponding to $v=7,8$ lead to above-the-barrier dissociation and have a large integrated width. They give rise to the broad $\mathrm{KE}$ bands at 0.18 and $0.3 \mathrm{eV}$, respectively. In contrast, the resonances adiabatically connected to the $v=9,10,11$ states are more of the Feshbach type and have an important VT component. As a consequence, the bands they give rise to are narrow and well resolved. The KE bands associated with these resonances are nonoverlapping, and this implies that their positions and widths do not vary with the delay $\tau$ when this is reduced from $\tau=561$ fs to $\tau=\tau_{0}$, as can be inferred from Eq. (23), with $\left[f_{v^{\prime}}^{(j)}\right]^{*}(E) f_{v}^{(j)}(E)=\left|f_{v}^{(j)}(E)\right|^{2} \delta_{v^{\prime} v}$. This invariance is clearly seen in panel (a) of Fig. 3, which shows how the various lines in the KE spectrum vary in amplitude with the delay $\tau$ for $\tau \in[-300 \mathrm{fs},+561 \mathrm{fs}]$.

Higher-energy resonances associated with $v>12$ are strongly of the Feshbach type, and the stronger VT effect prevailing in this range of energy is clearly observed in panel (c) of Fig. 3. Since populations trapped in these resonances supported basically by the upper adiabatic potential created at the one-photon avoided crossing [cf. Fig. 1(b)] cannot contribute to the fragment $\mathrm{KE}$ spectrum, the bands expected in the spectrum at these high energies, correlating with $v=$ $12,13,14$ on the $|u, n-1\rangle$ channel, are quenched (as compared to the bands associated with $v=10,11$, for example). Note that these bands do overlap each other to some extent. According to Eq. (23), these overlaps should give rise to interferences between these individual $v$-state signals in this range of energy. Close examination of the $\tau$ variations of these higher-energy bands associated with the $|u, n-1\rangle$ channel does confirm this. These variations are not seen in panel (a) of Fig. 3, as they are masked by the strong continuous bands depicting the variations of the intense peaks [shown by the black line in panel (c)] corresponding to the above-threshold dissociation (ATD) on the $|g, n-2\rangle$ channel, after a net absorption of two photons from $v=3,4$ states.

In the range $0-0.1 \mathrm{eV}$ of the $\mathrm{KE}$ spectrum of panel (c), a series of apparently well-resolved fine peaks is found. They arise from the interaction between overlapping resonances, in particular, those correlated to $v=5$ and $v=6$, both 
Stark-shifted downward ("pushed downward" by the opening barrier at the one-photon avoided crossing), the shift being normally stronger for $v=6$ than for $v=5$. This difference in the shifts, together with the fact that the width associated with $v=6$ is much broader than that of $v=5$, gives rise to a typical resonance overlapping situation. The fine structure of this lowenergy band indeed varies periodically with $\tau$, as expected for overlapping KE bands associated with overlapping resonances, giving rise to the interference pattern observed in this energy range in the KE spectrum of panel (a) of Fig. 3.

\section{Overlapping pump and probe pulses}

In all such situations (characterized by $\tau_{0}>\tau>-\tau_{0}$ ), the FC wave packet (the initial state) is instantly, i.e., nonadiabatically, projected onto Floquet resonances (shape and Feshbach) of $\mathrm{H}_{2}{ }^{+}, t_{0}$ corresponds to $\tau$, and

$$
Z_{\Psi_{0}}^{(\tilde{v})}=\sum_{v} c_{v}^{F C} Z_{\chi_{v}}^{(\tilde{v})} .
$$

As a consequence, the proton KE spectrum on channel $j=g$ or $u$, given by (here again, $t_{f}=\tau+\tau_{0}$ but $t_{0}=\tau$ )

$$
\begin{aligned}
S_{j}(E)= & \sum_{\tilde{v}} \sum_{\tilde{v}^{\prime}}\left[Z_{\Psi_{0}}^{(\tilde{v})}\right]^{*} Z_{\Psi_{0}}^{\left(\tilde{v}^{\prime}\right)} e^{-i / \hbar \int_{\tau}^{\tau+\tau_{0}} d t^{\prime}\left[\mathcal{E}_{\tilde{v}^{\prime}}\left(t^{\prime}\right)-\mathcal{E}_{\tilde{v}}\left(t^{\prime}\right)\right]} \\
& \times\left[f_{v^{\prime}}^{(j)}\right]^{*}(E) f_{v}^{(j)}(E),
\end{aligned}
$$

does not exhibit XUV-NIR delay-dependent interferences between overlapping resonance amplitudes. This is because the phase integral

$$
\begin{aligned}
& \int_{\tau}^{\tau+\tau_{0}} d t^{\prime}\left[\mathcal{E}_{\tilde{v}^{\prime}}\left(t^{\prime}\right)-\mathcal{E}_{\tilde{v}}\left(t^{\prime}\right)\right] \\
& \quad=\int_{0}^{\tau_{0}} d \theta\left[\mathcal{E}_{\tilde{v}^{\prime}}(\theta)-\mathcal{E}_{\tilde{v}}(\theta)\right], \quad\left(\theta=t^{\prime}-\tau\right)
\end{aligned}
$$

depends on the width of the NIR pulse (i.e., the total width $\approx 2 \tau_{0}$ ) and not on the XUV-NIR delay. The proton KE spectrum does depend on the XUV-NIR delay $\tau$, however, but continuously, through the quantities $Z_{\Psi_{0}}^{(\widetilde{v})}$ defined at $\tau$. This dependence is shown in panel (a) of Fig. 3 and can be understood by analyzing the extent to which the laser-induced resonances differ from the field-free $v$ states and how their positions and widths vary across the IR pulse.

With a delay $0 \mathrm{fs}<\tau=61 \mathrm{fs}<\tau_{0}$ [Fig. 2(c)], the XUV pump pulse precedes the maximum of the NIR pulse envelope. This means that the molecular system does experience a part of the pulse rise to the peak intensity. The shape resonances already come with non-negligible widths defined by the intensity of the NIR probe at this time. The closer $\tau$ is to zero, the more these resonance states will differ from the field-free vibrational states of the molecular ion and the projections of these $v$ states comprised in the initial FC wave packet onto resonances amount to a shake-up process: the initial FC state becomes an initial resonance wave packet. Once placed in this superposition of resonance states, the system sees the remaining part of the intensity profile of the NIR pulse, which, being relatively slow, ensures an adiabatic evolution of the resonances under this pulse. During the rise of the NIR envelope, the ion experiences increasing field amplitude in time and sees a lowering of the lower adiabatic potential barrier and a widening of the potential energy gap at the one-photon avoided crossing. All the resonances onto which the $v>8$ field-free vibrational states project are now above-the-barrier shape resonances. The width $\Gamma$ of these shape resonances, already important at the time of birth of the resonance wave packet, further increases, and the more so the higher-lying the resonances are with respect to the potential energy barrier at the one-photon crossing. The broadening and the overlap of these resonances are sufficient to wash out the structures previously found in the higher-energy wing of the KER spectrum associated with the $|u, n-1\rangle$ channel, giving the unresolved band as seen in Fig. 2(c).

On the $|g, n-2\rangle$ channel, we observe essentially the same well-resolved peaks as found in the case of an adiabatic resonance branching, showing that the dissociation dynamics of the $v=2,3,4$ components via the two-photon channels is not much affected by the movements of the dissociation barrier defined by the one-photon avoided crossing.

The situation is not much different for $\tau \leqslant 0$ fs [Figs. 2(d) and 2(e)], corresponding to preparing the molecular ion at or after the center of the NIR pulse envelope, where the peak intensity is attained and the potential energy barrier (at the one-photon crossing) is lowest. Once again, the shake-up-type projection of the initial FC wave packet on the high-intensity resonances is sudden (strongly nonadiabatic), but subsequent resonance transport is adiabatic. As the potential barrier is rising afterwards, less time is offered for dissociation through low-energy shape resonances and one observes a decrease, even an extinction for $\tau<0$ fs, of the amplitudes of low-kinetic-energy peaks in the spectrum associated with the $|u, n-1\rangle$ channel. The same holds true for peaks corresponding to dissociation on the $|g, n-2\rangle$ channel. The global effect of all the above effects is to produce a shift of the center of gravity of the distribution towards higher kinetic energies when $\tau$ decreases past zero.

\section{B. Short and ultrashort probe pulses: Nonadiabatic dynamics}

\section{Short NIRS probe pulse}

The case of the shorter probe pulse, $T_{\mathrm{FWHM}}=35 \mathrm{fs}$, is illustrated in Fig. 4 (organized in the same manner as Fig. 2) and Fig. 5, which shows the continuous variation of the KE spectrum as a function of the delay $\tau$. Here, the evolution of the Floquet resonances under the NIR probe is expected to be less adiabatic than in the previous case of the $T_{\text {FWHM }}=150 \mathrm{fs}$ pulse. Nonadiabatic transitions are expected to occur in the vicinity of the peak of the pulse, where the slope toward the peak intensity is steepest. For $\tau>\tau_{0} \approx 50 \mathrm{fs}$, the Floquet resonances are accessed adiabatically and may evolve adiabatically during the slow rise of the pulse, to quickly mix with each other nonadiabatically just before reaching $t \simeq \tau$, i.e., the center of the pulse. These nonadiabatic transitions in time between the Floquet resonances cause a loss of resolution in the kinetic energy spectrum [as seen Fig. 4(b)] in comparison with what was found in the case of the longer pulse [Fig. 2(b)]. Also, because of these nonadiabatic transitions, Eq. (22) is no longer valid. To appreciate how nonadiabatic effects would modify this expression, let us imagine that nonadiabatic transitions between instantaneous Floquet eigenstates occur at $t=\bar{t}<\tau$ (some time on the steep rise towards the center of 


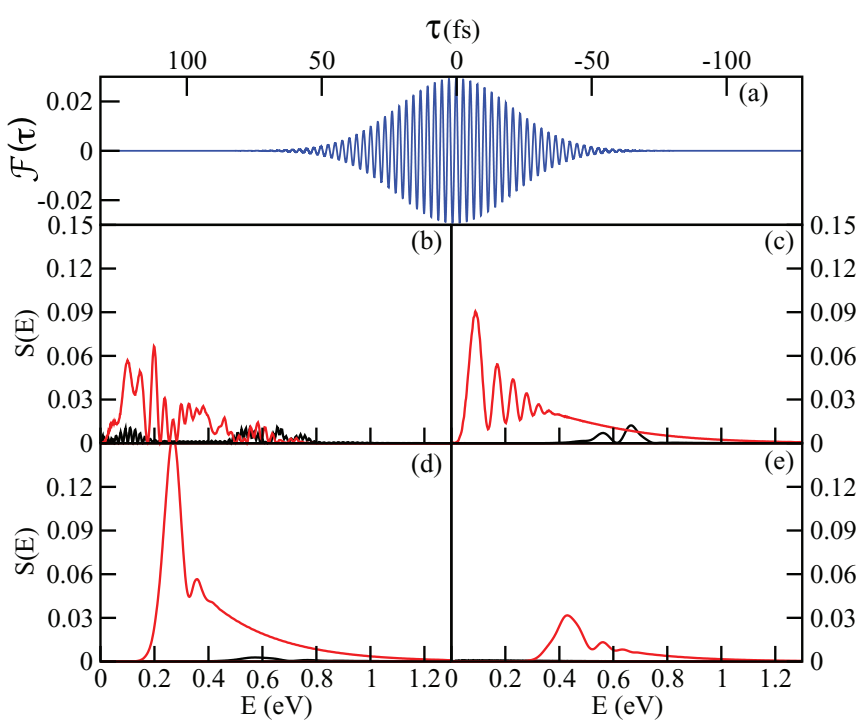

FIG. 4. (Color online) Same as for Fig. 2, but for a $T_{\mathrm{FWHM}}=35 \mathrm{fs}$ NIR probe pulse, shown in panel (a). In panel (b), $\tau=+132 \mathrm{fs}$; in panel (c), $\tau=+32 \mathrm{fs}$; in panel (d), $\tau=0 \mathrm{fs}$; and in panel (e), $\tau=-28 \mathrm{fs}$.

the pulse) and can be described by a transition matrix $\mathbb{Z}^{N A}$, of elements $Z_{\tilde{v}, r}^{N A}$, and that each resonance $\left|\Psi^{(r)}(R, t)\right\rangle$ evolves afterwards adiabatically to yield a KE distribution amplitude $f_{r}^{(j)}(E)$ on each channel $j$. Then, the KE amplitude, on a given channel, resulting from the complete time evolution of the initial FC wave packet would read

$$
f^{(j)}(E)=\sum_{v} c_{v}^{F C} e^{-i / \hbar\left[\epsilon_{v}\left(\tau-\tau_{0}\right)+\int_{\tau-\tau_{0}}^{\bar{\tau}} d t^{\prime} \mathcal{E}_{v}\left(t^{\prime}\right)\right]} \sum_{r} Z_{v, r}^{N A} f_{r}^{(j)}(E) .
$$

Taking the squared modulus of this, we get the expected $\mathrm{KE}$ distribution. Consider the particular case where the distribution amplitudes $f_{r}^{(j)}(E)$ do not overlap each other, $\left[f_{r}^{(j)}(E)\right]^{*} f_{r^{\prime}}^{(j)}(E)=\left|f_{r}^{(j)}(E)\right|^{2} \delta_{r, r^{\prime}}$, for which an adiabatic Floquet state transport throughout the pulse would give a delay-independent KE spectrum. In the present case of strong

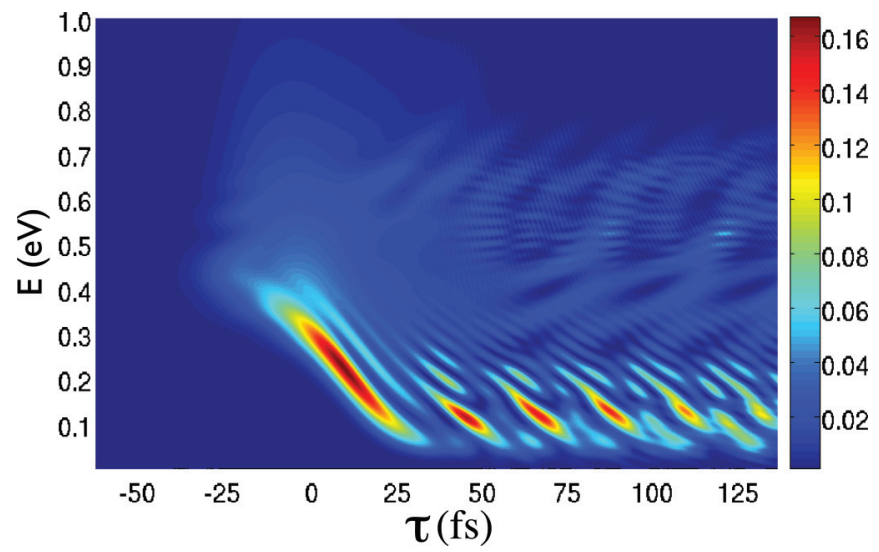

FIG. 5. (Color online) Fragment relative KE spectrum viewed as a function of the delay $\tau$ between the XUV pump pulse and the $T_{\text {FWHM }}=35 \mathrm{fs}$ NIR probe pulse. The delay scale is as defined in panel (a) of Fig. 4. nonadiabaticity, from Eq. (27) we have

$$
\begin{gathered}
S^{(j)}(E)=\sum_{r} A(r, \tau)\left|f_{r}^{(j)}(E)\right|^{2}, \\
A(r, \tau)=\sum_{v} \sum_{v^{\prime}}\left(c_{v}^{F C} Z_{v, r}^{N A}\right)^{*}\left(c_{v^{\prime}}^{F C} Z_{v^{\prime}, r}^{N A}\right) \\
\quad \times e^{\left.-i / \hbar\left(\epsilon_{v^{\prime}}-\epsilon_{v}\right)\left(\tau-\tau_{0}\right)+\int_{\tau-\tau_{0}}^{\bar{t}} d t^{\prime} \mathcal{E}_{v^{\prime}}\left(t^{\prime}\right)-\mathcal{E}_{v}\left(t^{\prime}\right)\right]},
\end{gathered}
$$

which is strongly $\tau$ dependent, the function $A(r, \tau)$ modulating the individual resonance (labeled $r$ ) signals by a periodic function, giving rise to periodic depletions and enhancements of the signals, i.e., a $\tau$-dependent interference pattern. This is seen in Fig. 5 for $\tau \gtrsim 35$ fs.

For $\tau_{0}>\tau>-\tau_{0}$ the preparation of the Floquet resonance wave packet is sudden. What was said previously about the effects of the variations of the widths of the resonances across the intensity range covered by the pulse width, in the equivalent situations but in the longer NIR pulse case, applies here also. In addition, the nonadiabatic transitions between certain resonances occurring in the vicinity of the pulse's peak causes a quenching in the two-photon signals on the channel $|g, n-2\rangle$ and in the low-energy KE signals (those corresponding to $v=$ $5,6)$ earlier than in the longer $\left(T_{\mathrm{FWHM}}=150 \mathrm{fs}\right)$ pulse. [The spectra in panels (c) and (d) of Fig. 4 are comparable in form to those in panels (d) and (e), respectively, of Fig. 2.] The overall blueshift of the center of gravity of the KE spectrum occurs already at the maximum of the NIR pulse in the present case $\left(T_{\mathrm{FWHM}}=35 \mathrm{fs}\right)$, i.e., earlier than in the $T_{\mathrm{FWHM}}=150 \mathrm{fs}$ pulse. These marks of nonadiabaticity observed in the present case can also be expressed differently, namely, that the rapid rise of the dissociation barrier accompanying the rapid variation of the intensity profile (after reaching the peak intensity) largely reduces the dissociation probability on the $|u, n-1\rangle$ channel from the low-energy states, even at $\tau=0 \mathrm{fs}$. The rapid fall of the intensity after its peak also limits the two-photon (higher-order) process and causes a decrease in the dissociation probability on the $|g, n-2\rangle$ channel, as reflected in the weaker intensities found [in Fig. 4(c) for the case of the $T_{\mathrm{FWHM}}=35 \mathrm{fs}$ probe pulse] for all the bands associated with this channel as compared to the case of the $T_{\mathrm{FWHM}}=150 \mathrm{fs}$ pulse of Fig. 2(c).

\section{Ultrashort probe pulse}

With an even shorter, $T_{\mathrm{FWHM}}=7 \mathrm{fs}$ NIR probe pulse, nonadiabatic dynamics effects are stronger and set in almost right at the onset of the pulse, and $\tau_{0}$ is so short that the phase integral of the complex resonance energy appearing in Eqs. (27) and (28), even more appropriate in this strong nonadiabaticity case, is negligible as compared to $\left(\epsilon_{v}-\epsilon_{v^{\prime}}\right)\left(\tau-\tau_{0}\right)$ so that the beating patterns observed in the KE energy spectrum at $\tau>\tau_{0}(>0)$ in Fig. 6(b) reflect the coherence of the initial FC field-free vibrational wave packets. Thus, if attention is focused only on the range of $\tau$ denoting nonoverlapping XUV-NIR pulses situations, then the 7-fs NIR pulse appears to only serve to open, almost instantly, a gate to dissociation with which the incoming wave packet is synchronized to give an efficient dissociation or its quenching (the molecule hits or misses that gate), depending on the value of $\tau$ [14]. The interference pattern in the spectrum gives information only on the initial vibrational wave packet formed as the ion was 


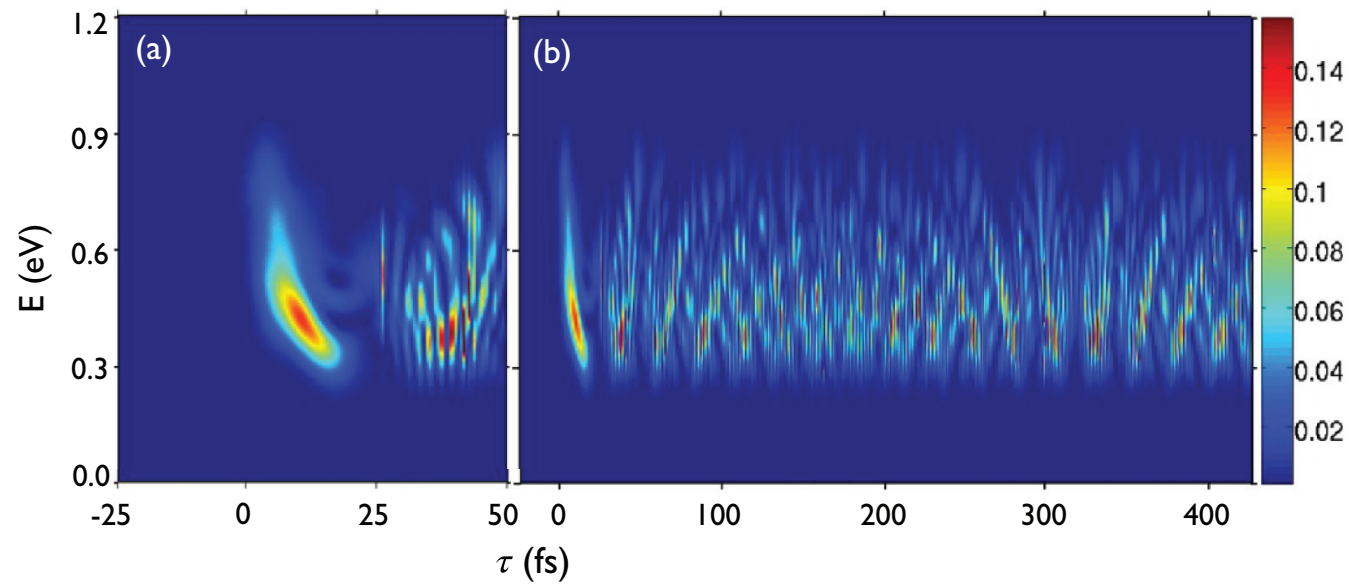

FIG. 6. (Color online) Fragment relative KE spectrum viewed as a function of the delay $\tau$ between the XUV pump pulse and the $T_{\mathrm{FWHM}}=7 \mathrm{fs}$ NIR probe pulse. The delay $\tau$ ranges from $-25 \mathrm{fs}$ to $+400 \mathrm{fs}$ in panel (b). Panel (a) is an enlargement of the region $\tau \in[-25 \mathrm{fs},+25 \mathrm{fs}]$, made to show the smooth variation of the KE spectrum with $\tau$ in the pump-probe pulse overlap condition.

born under the XUV pulse. This is one of the conclusions reached in Ref. [10], where this interference pattern, also found in the experimental spectrum to be in complete agreement with the theoretical one, was resolved by Fourier transform techniques and was shown to result from the beatings of signals bearing the signatures of vibrational states composing the initial Franck-Condon wave packet. In fact, the 7-fs FWHM pulse does more than just open an instant gate to dissociation. The part of the $\tau$-dependent spectrum associated with the XUV-NIR overlapping conditions [Fig. 6(a)] again gives a different, interference-free behavior, with the same characteristic blueshift of the center of gravity of the spectrum as found in the longer-pulse cases (albeit occurring faster here than in these longer cases).

\section{Vibrational shake-up}

It is interesting to follow the branching of vibrational states onto resonances and their gradual deformations within the intensity profile of the NIR field. To this end, we repeat the propagation procedure for a fixed value of the delay $\tau$ between the pump and the probe pulses, but this time starting from initial states that are vibrational states $\varphi_{v}(R)=\langle R \mid v\rangle$ of the ground-state molecular ion, i.e., eigenstates of $T_{N}+V_{1}(R)$. From the results of these calculations we can calculate the projection of $\chi_{0}^{\mathrm{H}_{2}}$, the vibrational ground state of $\mathrm{H}_{2}$, onto the $|g\rangle$ component $\varphi_{v}^{g}(R, t)$ of the propagated state $\varphi_{v}(R, t)=$ $U(t, 0) \varphi_{v}(R)$ :

$$
P_{v}(t)=\frac{\left|\left\langle\chi_{0}^{\mathrm{H}_{2}} \mid \varphi_{v}^{g}(t)\right\rangle\right|^{2}}{\left\|\varphi_{v}(t)\right\|^{2}} .
$$

For times $t$ falling within the width of the NIR pulse centered at $t=\tau$, this represents, in the adiabatic Floquet limit, the projection of $\chi_{0}^{\mathrm{H}_{2}}$ onto the instantaneous (time-parametrized) resonance state that correlates at zero field to the molecular vibrational state $|v\rangle$. In contrast, at any time preceding the onset of the NIR pulse, $P_{v}$ is nothing else than the Franck-Condon ionization profile. Its deviation from the Franck-Condon value after the onset of the NIR pulse thus reveals precisely the difference between field-free vibrational states and resonances and the dynamics followed by these resonances.

In the case of $T_{\mathrm{FWHM}}=35 \mathrm{fs}$ NIR probe pulse, Fig. 7 shows the evolution of the projection $P_{v}$ for $v=7,8,9$. Two observations can be made: First, within the NIR pulse, $P_{v}$ oscillates at a high frequency, actually equal to $2 \omega$. These then disappear at the end of the pulse and are replaced by much slower ones. Second, shortly after the NIR pulse is encountered, $P_{v}$ tends to increase in the mean (within the $2 \omega$ oscillations described above) before decaying, the increase being most important, and setting in earliest, in the case $v=9$, and least important in

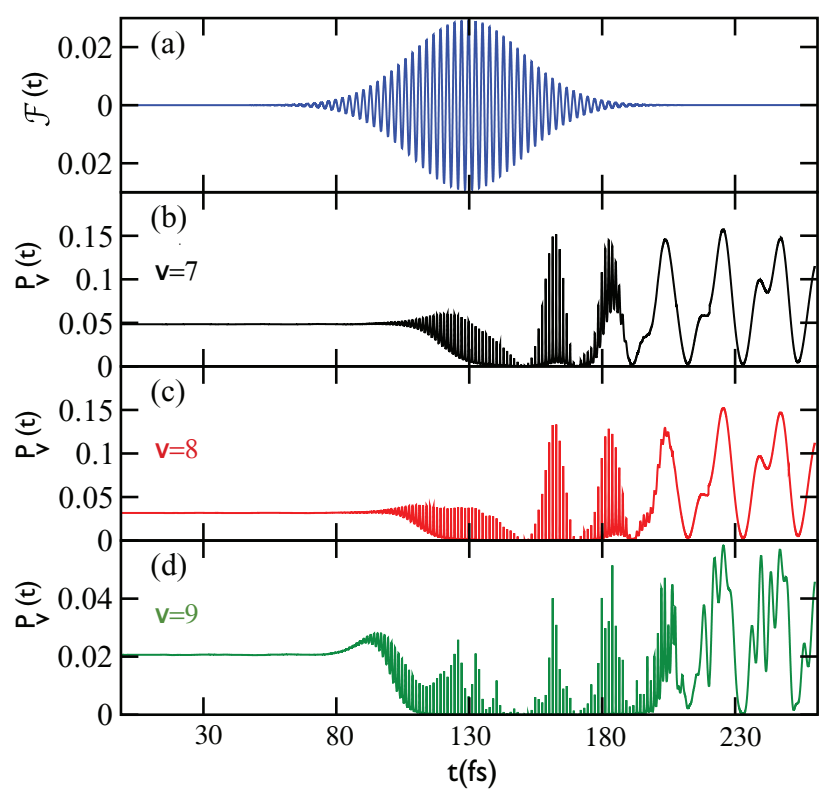

FIG. 7. (Color online) Time evolution of the projection $P_{v}$, calculated using Eq. (30), of the FC wave packet $\chi_{0}^{\mathrm{H}_{2}}$ onto the individual $v$ states of the ion, which are themselves transported from an initial time $t=0$ onto resonances defined by the NIR pulse at a time $t$. Panel (a) shows the $T_{\mathrm{FWHM}}=35 \mathrm{fs}$ NIR probe pulse centered at $t=130 \mathrm{fs}$. $v=7$ in panel (b), $v=8$ in panel (c), and $v=9$ in panel (d). 
the $v=8$ case. Though limited to those values of $v$ in the high end of the initial FC vibrational profile, this observation is a confirmation of the hypothesis of a vibrational shake-up within the width of the intense NIR pulse, in favor of resonances correlating with high-energy vibrational states.

The $2 \omega$ oscillations arise from the beating of Floquet components of the time-dependent wave packet with the given $v$ as the initial condition. These components correspond to some Floquet eigenstates that belong to a Brillouin zone nearby and distant by $2 \omega$ from the one containing the main resonance that adiabatically connects with the field-free $v$ state at low intensity. These components are acquired by the time nonadiabatic couplings between the time-parametrized Floquet states, which occur only when the field intensity is sufficiently high to enable second-order, two-photon processes. Postpulse, slow-frequency oscillations denote beating of the amplitude of the initial $v$ state (the dominant one) with signals associated with new field-free vibrational components. These correspond to Floquet resonances that are mixed in (at about midpulse) with those correlated to the initial $v$ state through the same nonadiabatic couplings mentioned above in relation to the $2 \omega$ oscillations. In the cases $v=7,8$, the postpulse oscillations denote the beating of two frequencies, implying that two other $v$ states are comprised in the final wave packet and were accessed, during the pulse, by these nonadiabatic transitions. For $v=9$, many more vibrational states seem to have been populated by the same mechanism by the end of the pulse.

\section{CONCLUSION}

We have addressed in this paper a number of important issues concerning the manifestations of the dynamical implications of dressed transition states in strong-field molecular fragmentation, induced and probed by a NIR pulse. We show how the variations of this observable, the KER spectrum of molecular fragments, as a function of the time delay between this probe pulse and an XUV ionizing pump pulse carry rich information regarding the way dressed transition states, laser-induced resonances, are accessed and used in the NIR laser-driven dynamics of the molecule on its way towards complete dissociation. The discussion is centered on the distinction between adiabatic versus nonadiabatic behaviors of the molecule. Two levels of adiabaticity in time are to be distinguished: one with respect to the preparation of the wave packet, which is controlled by the pump-probe pulse delay, and another one with respect to the dynamics of the Floquet resonances, the dressed transition states of the molecular dissociation process per se, which strongly depends on the duration of the NIR pulse.

Within a very long NIR pulse (the 150-fs FWHM pulse in the above is representative of this case), the Floquet resonances are transported adiabatically from one time to another, and their connection with field-free vibrational states can be followed smoothly in time. For a time delay denoting an XUV pump pulse preceding the NIR pulse, or overlapping this pulse near its onset, each $v$ component of the initial wave packet is slowly transported onto a single Floquet resonance, i.e., one has a one-to-one correspondence between the field-free vibrational states and the laser-induced resonances. This is the signature of adiabatic behavior. More precisely, it can be shown that adiabaticity is basically governed by two key factors, namely, a slow variation in time of the moleculefield coupling, which guarantees a small time derivative of this coupling [42], and well-separated resonances (i.e., with distinct energy positions and widths) [34,37]. For situations where the pump-probe pulse overlap occurs at a time closer to the NIR pulse's peak, the Floquet resonances defined by the field intensity of the NIR probe pulse at this time may differ strongly, in every respect (position, width, nodal properties), from the field-free vibrational states, and these strong-field dressed transition states are accessed nonadiabatically and in a many-to-one fashion (i.e., a given $v$ may correlate to several Floquet resonances). This actually is a consequence of the sudden strong NIR field intensity which is felt by the molecular ion as prepared by the XUV pulse. However, the subsequent dynamics of these resonances unfold adiabatically, i.e., there is no mixing between them, and they should connect back to the corresponding field-free $v$ state at the end of the NIR pulse, except if resonance quasidegeneracies (overlaps) are dynamically encountered at some time during this long pulse. Though nonadiabatic couplings between the (slowly time-varying) Floquet states are weak, they can nevertheless become effective when this quasidegeneracy occurs, resulting in a final vibrational wave packet that is different from the initial one. In an analysis of the same type as done in Fig. 7, but for the longer (150-fs FWHM) NIR pulse, during which the Floquet state transport is normally adiabatic, postpulse oscillations were observed in some $P_{v}$ (results not shown here) and indicate nonadiabatic transitions that result from quasidegeneracy situations of this type.

Under a shorter NIR pulse, high-intensity Floquet resonances are accessed nonadiabatically and at an earlier time relative to the NIR field period; thus the pulse is shorter, causing an early vibrational shake-up effect. The Floquet states themselves evolve nonadiabatically, i.e., strong nonadiabatic transitions between them occur as the system sweeps through this short, intense pulse. The strong nonadiabaticity is signaled by a modulation of features of the KER spectrum by a delay-dependent interference pattern for values of the delay denoting a pump pulse preceding the probe pulse. The effect is more pronounced the shorter the NIR pulse. In the case of the shortest pulse considered ( 7 fs FWHM), nonadiabatic mixing of the Floquet states is so strong that this modulation affects all the lines of the KER spectrum, and the interference pattern observed in this range of the pump-probe delay merely reflects the coherence of the initial wave packet. This can actually be used to image this initial field-free $\mathrm{H}_{2}{ }^{+}$vibrational wave packet prepared by the XUV attosecond pump pulse. Insofar as this wave packet of the molecular ion constitutes by itself the first transition state of the dissociative ionization of the neutral parent molecule $\left(\mathrm{H}_{2}\right)$, this imaging objective is already of great interest, as emphasized in Ref. [10]. There is more to this ultrafast pump-probe experiment, however, as the dynamics that unfold under the XUV-NIR pulse overlap conditions also gives in all cases an interference-free delay dependance of the KER spectrum. This part of the delay-dependent spectrum shows in all cases, almost irrespective of the duration of the NIR pulse, qualitatively the same characteristic blueshift of the whole KER spectrum as the pump pulse is swept across the probe pulse. It carries the dynamic signature of the 
resonances, laser-dressed transition states encountered during the laser-induced molecular fragmentation process. In addition to the implications of the present analysis pertaining to an imaging objective, that of a laser-dressed transition state, which is nontrivial by itself, we should also mention its obvious application in quantum control. Molecular dissociation and final vibrational distributions can indeed be controlled through an interplay of Feshbach and shape resonances that can be followed through the delay-dependent fragments' KER spectrum, and tuned by the intensity and duration of the probe pulse.

\section{ACKNOWLEDGMENTS}

The authors acknowledge fruitful discussions with Prof. Arne Keller and also support from the European Union (Project No. ITN-2010-264951, CORINF). T.T.N.D. and C.L. acknowledge the Natural Sciences and Engineering Research Council of Canada (NSERC) for financial support of this work over the years. The computational resources of Compute Canada (CLUMEQ) have also been partly used in this work.
[1] A. Zewail, J. Phys. Chem. 104, 5660 (2000).

[2] C. Petersen, E. Péronne, J. Thogersen, H. Stapelfeldt, and M. Machholm, Phys. Rev. A 70, 033404 (2004).

[3] C. Trump, H. Rottke, and W. Sandner, Phys. Rev. A 59, 2858 (1999).

[4] T. Ergler, A. Rudenko, B. Feuerstein, K. Zrost, C. D. Schröter, R. Moshammer, and J. Ullrich, Phys. Rev. Lett. 97, 193001 (2006).

[5] P. B. Corkum, Phys. Rev. Lett. 71, 1994 (1993).

[6] M. Drescher, M. Hentschel, R. Kienberger, M. Uiberacker, V. Yakovlev, A. Scrinzi, T. Westerwalbesloh, U. Kleineberg, U. Heinzmann, and F. Krausz, Nature (London) 419, 803 (2002).

[7] E. Goulielmakis, Z.-H. Loh, A. Wirth, R. Santra, N. Rohringer, V. Yakosvlev, S. Zherebstov, T. Pfeifer, A. M. Azzeer, M. F. Kling, S. R. Leone, and F. Krausz, Nature (London) 466, 739 (2010).

[8] A. D. Bandrauk and M. Ivanov, Quantum Dynamic Imaging (Springer-Verlag, New York, 2011).

[9] T. Remetter, P. Johnsson, J. Mauritsson, K. Varju, Y. Ni, F. Lépine, E. Gustafsson, M. F. Kling, J. Khan, R. LópezMartens, K. J. Schafer, M. J. J. Vrakking, and A. L'Huillier, Nat. Phys. 2, 323 (2006).

[10] F. Kelkensberg, C. Lefebvre, W. Siu, O. Ghafur, T. T. NguyenDang, O. Atabek, A. Keller, V. Serov, P. Johnsson, M. Swoboda, T. Remetter, A. LHuillier, S. Zherebtsov, G. Sansone, E. Benedetti, F. Ferrari, M. Nisoli, F. Lepine, M. F. Kling, and M. J. J. Vrakking, Phys. Rev. Lett. 103, 123005 (2009).

[11] C. Lefebvre, Ph.D. thesis, Université Laval and Université ParisSud 11, 2008.

[12] F. He and U. Thumm, Phys. Rev. A 81, 053413 (2010).

[13] A. D. Bandrauk, S. Barmaki, and G. L. Kamta, Phys. Rev. Lett. 98, 013001 (2007).

[14] C. Châteauneuf, T. T. Nguyen-Dang, N. Ouellet, and O. Atabek, J. Chem. Phys. 108, 3974 (1998).

[15] J. J. Hua and B. D. Esry, Phys. Rev. A 80, 013413 (2009).

[16] O. Atabek, R. Lefebvre, and T. T. Nguyen-Dang, in Handbook of Numerical Analysis (Elsevier, New York, 2003).
[17] V. N. Serov, A. Keller, O. Atabek, and N. Billy, Phys. Rev. A 68, 053401 (2003).

[18] M. D. Feit, J. A. Fleck, and A. Steiger, J. Comp. Phys. 47, 412 (1982).

[19] A. Keller, Phys. Rev. A 52, 1450 (1995).

[20] R. Heather and H. Metiu, J. Chem. Phys. 86, 5009 (1987).

[21] G. Floquet, Ann. Ec. Norm. Supér. 12, 47 (1883).

[22] H. Sambe, Phys. Rev. A 7, 2203 (1973).

[23] J. H. Shirley, Phys. Rev. B 138, B979 (1965).

[24] S. Guérin and H. R. Jauslin, Adv. Chem. Phys. 125, 147 (2003).

[25] S. C. Althorpe, D. J. Kouri, D. Hoffman, and N. Moiseyev, Chem. Phys. 217, 289 (1997).

[26] N. Moiseyev, Phys. Reports 302, 212 (1998).

[27] O. Atabek, R. Lefebvre, C. Lefebvre, and T. T. Nguyen-Dang, Phys. Rev. A 77, 043413 (2008).

[28] A. Giusti-Suzor and F. H. Mies, Phys. Rev. Lett. 68, 3869 (1992).

[29] A. F. J. Siegert, Phys. Rev. 56, 750 (1939).

[30] C. Lefebvre, T. T. Nguyen-Dang, and O. Atabek, Phys. Rev. A 75, 023404 (2007).

[31] O. Atabek, R. Lefebvre, and T. T. Nguyen-Dang, Adv. Quant. Chem. 60, 51 (2010).

[32] J. M. Okuniewicz, J. Math. Phys. 15, 1587 (1974).

[33] T. Kato, J. Phys. Soc. Jpn. 5, 435 (1950).

[34] M. S. Sarandy and D. A. Lidar, Phys. Rev. A 71, 012331 (2005).

[35] H. P. Breuer, K. Dietz, and M. Holthaus, Phys. Rev. A 47, 725 (1993).

[36] T. T. Nguyen-Dang, E. Sinelnikov, A. Keller, and O. Atabek, Phys. Rev. A 76, 052118 (2007).

[37] A. Fleischer and N. Moiseyev, Phys. Rev. A 72, 032103 (2005).

[38] M. V. Berry, Proc. R. Soc. A 392, 45 (1984).

[39] R. Lefebvre, O. Atabek, M. Sindelka, and N. Moiseyev, Phys. Rev. Lett. 103, 123003 (2009).

[40] O. Atabek, R. Lefebvre, M. Lepers, A. Jaouadi, O. Dulieu, and V. Kokoouline, Phys. Rev. Lett. 106, 173002 (2011).

[41] M. Chrysos, O. Atabek, and R. Lefebvre, Phys. Rev. A 48, 3855 (1993).

[42] A. Jaouadi, M. Desouter-Lecomte, R. Lefebvre, and O. Atabek, J. Phys. B 46, 145402 (2013). 